\title{
¿LA TECNOLOGÍA DE LA INTELIGENCIA ARTIFICIAL (IA) PUEDE PERJUDICAR O FAVORECER A LOS CONSUMIDORES?
}

\author{
Luis González Vaqué ${ }^{1}$ \\ International Association of Consumer Law (IACL) \\ Ex-Consejero de la Dirección General de Mercado Interior de la Comisión Europea
}

Title: Is Artificial Intelligence (AI) Technology Disadvantageous or Beneficial for Consumers?

Resumen: La inteligencia artificial (IA) puede ayudar a encontrar soluciones a muchos de los problemas de nuestra sociedad. Esto solo puede lograrse si la tecnología es de gran calidad y se desarrolla y se utiliza de tal manera que merezca la confianza de los ciudadanos. Por lo tanto, un marco estratégico de la UE basado en los valores de la Unión ofrecerá a los ciudadanos la confianza necesaria para que acepten las soluciones basadas en la IA, animando al mismo tiempo a las empresas a desarrollarlas y utilizarlas.

Un Reglamento sobre un enfoque europeo respecto a la inteligencia artificial y la actualización del plan coordinado sobre la IA garantizarán la seguridad y los derechos fundamentales de las personas y las empresas, reforzando al mismo tiempo la inversión y la innovación en todos los países de la UE. En este artículo se analiza si su adopción será beneficiosa para los consumidores.

Palabras clave: Unión Europea, Inteligencia Artificial, Programas informáticos, Protección de los consumidores, Mercado interior, Desarrollo empresarial, Almacenamiento de datos personales.

Abstract: Artificial intelligence (AI) can help find solutions to many of society's problems. This can only be achieved if the technology is of high quality, and developed and used in ways that earns peoples' trust. Therefore, an EU strategic

\footnotetext{
${ }^{1}$ Dirección electrónica: gonzalu70 [at] outlook.es
} 
framework based on EU values will give citizens the confidence to accept AI-based solutions, while encouraging businesses to develop and deploy them.

A new Regulation on a European Approach for Artificial Intelligence and the update of the Coordinated Plan on AI will guarantee the safety and fundamental rights of people and businesses, while strengthening investment and innovation across EU countries. This article looks at whether its adoption will be beneficial to consumers.

Key words: European Union, Artificial Intelligence, Software, Consumer protection, Internal market, Business development, Personal data storage

Sumario: 1. Introducción: ¿Qué es la "Inteligencia Artificial"? 2. Estrategia europea sobre la IA y el Libro Blanco de 2020. 3. La Propuesta de Reglamento sobre la IA. 4. Conclusión: 'fumus boni iuris'. 5. Bibliografía.

\section{Introducción: ¿Qué es la "Inteligencia Artificial"?}

Según la Comisión Europea la inteligencia artificial (IA) puede ayudar a encontrar soluciones a muchos de los problemas de nuestra sociedad: obviamente esto solo puede lograrse si la tecnología es de gran calidad y se desarrolla y se utiliza de tal manera que merezca la confianza de los ciudadanos ${ }^{2}$. Por lo tanto, siempre según la misma fuente, un marco estratégico de la UE basado en los valores de la Unión permitirá ofrecer a los ciudadanos la confianza necesaria para que acepten las soluciones basadas en la IA, incitando al mismo tiempo a las empresas a desarrollarlas y utilizarlas.

El objetivo del presente artículo es reflexionar sobre las ventajas e inconvenientes que la aplicación de esta nueva tecnología puede tener para los consumidores. Nuestra reflexión se basará en el conjunto de acciones proyectadas por la propia Comisión para impulsar la excelencia en la IA, así como en las normativas para garantizar que la tecnología en cuestión sea fiable. Más concretamente, nos referiremos a una reciente propuesta de Reglamento sobre un enfoque europeo respecto a la inteligencia artificial (en lo sucesivo la "Propuesta de Reglamento sobre la $\left.\mathrm{IA}^{\prime \prime}\right)^{3}$. En principio, esta nueva normativa comunitaria tendrá por objeto la actualización del plan coordinado sobre la IA a fin de garantizar la seguridad y los derechos fundamentales de las personas y las empresas, reforzando al mismo tiempo la inversión y la innovación en todos los países de la UE.

\footnotetext{
${ }^{2}$ https://ec.europa.eu/info/strategy/priorities-2019-2024/europe-fit-digital-age/excellence-trustartificial-intelligence_es

3 Proposal for a Regulation of the European Parliament and of the Council Laying Down Harmonised Rules On Artificial Intelligence (Artificial Intelligence Act) and Amending Certain Union Legislative Acts \{SEC(2021) 167 final $\}$ - \{SWD(2021) 84 final $\}$ - \{SWD(2021) 85 final $\}$ - Documento COM(2021) 206 final 2021/0106 de 21.4.2021, de la cual no existe una versión Española, cuando redactamos el presente artículo (por ello todas las citas textuales del mismo corresponden a traducciones libres del autor).
} 
No podemos seguir adelante sin definir qué se entiende por IA. Por supuesto, tenemos que recurrir en primer lugar a la noción que figura en la Propuesta de Reglamento sobre la IA; subrayaremos en este contexto lo que se indica en el sexto considerando del texto que se propone:

«La noción de sistema de inteligencia artificial debe definirse claramente para garantizar la seguridad jurídica y, al mismo tiempo, proporcionar la flexibilidad necesaria para adaptarse a futuros desarrollos tecnológicos. La definición debe basarse en las características funcionales clave del software, en particular la capacidad, para un conjunto dado de objetivos definidos por seres humanos, de generar resultados como contenido, predicciones, recomendaciones o decisiones que influyen en el entorno con el que el sistema interactúa, ya sea en una dimensión física o digital. Los sistemas de IA pueden diseñarse para funcionar con distintos niveles de autonomía y utilizarse de forma independiente o como componente de un producto, independientemente de si el sistema está integrado físicamente en el producto (integrado) o sirve a la funcionalidad del producto. sin estar integrado en el mismo (no incluido). La definición de sistema de IA debe complementarse con una lista de técnicas y enfoques específicos utilizados para su desarrollo, que deben mantenerse actualizados a la luz de los desarrollos tecnológicos y del mercado mediante la adopción de actos delegados por parte de la Comisión para modificar esa lista.».

Más adelante, en el artículo 3.1, el sistema de AI se define como «software que se desarrolla con una o más de las técnicas y enfoques enumerados en el Anexo $\mathrm{I}^{4}$ y puede, para un conjunto dado de objetivos definidos por seres humanos, generar resultados como contenido, predicciones, recomendaciones o decisiones que influyen en los entornos con los que interactúan».

Cabe señalar que, en abril de 2018, en la Comunicación de la Comisión "Inteligencia artificial para Europa"5, se especificaba ya que «el término 'inteligencia artificial' (IA) se aplica a los sistemas que manifiestan un comportamiento inteligente, pues son capaces de analizar su entorno y pasar a la acción -con cierto grado de autonomíacon el fin de alcanzar objetivos específicos»y, acto seguido, se aclaraba que «los sistemas basados en la IA pueden consistir simplemente en un programa informático (p. ej. asistentes de voz, programas de análisis de imágenes, motores de búsqueda, sistemas de reconocimiento facial y de voz), pero la IA también puede estar incorporada en dispositivos de hardware ( $p$. ej. robots avanzados, automóviles autónomos, drones o aplicaciones del internet de las cosas)».

\footnotetext{
${ }^{4}$ Relativo a "Artificial Intelligence Techniques and Approaches referred to in Article 3, point 1: (a) Machine learning approaches, including supervised, unsupervised and reinforcement learning, using a wide variety of methods including deep learning; (b) Logic- and knowledge-based approaches, including knowledge representation, inductive (logic) programming, knowledge bases, inference and deductive engines, (symbolic) reasoning and expert systems; [and] (c) Statistical approaches, Bayesian estimation, search and optimization methods. ${ }^{5}$ COM (2018) 237 final: https://eur-lex.europa.eu/legal-content/ES/TXT/?uri=COM\%3A2018\%3A237\%3AFIN
} 
Ciertamente, otras definiciones pueden servirnos para complementar una mejor percepción del concepto, como, por ejemplo, que la IA es «la habilidad de los ordenadores para hacer actividades que normalmente requieren inteligencia humana» 6 ; o «la capacidad de las máquinas para usar algoritmos, aprender de los datos y utilizar lo aprendido en la toma de decisiones tal y como lo haría un ser humano ${ }^{\mathbf{7}}$. Por otra parte, dada su creciente importancia, no es de extrañar que el Diccionario de la RAE incluya también la correspondiente definición de la AI:

«Disciplina científica que se ocupa de crear programas informáticos que ejecutan operaciones comparables a las que realiza la mente humana, como el aprendizaje o el razonamiento lógico».

Pero no pretendemos enumerar aquí todas las diversas definiciones que se han publicado; de todos modos, resulta hasta cierto punto sorprendente que éstas, pese a ser numerosas y diversas, coincidan fundamentalmente en sus elementos más sustanciales y en la descripción del concepto del que se trata ${ }^{\mathbf{8}}$. Por ello pasaremos seguidamente a exponer los antecedentes del documento objeto de nuestras reflexiones.

\section{Estrategia europea sobre la IA y el Libro Blanco de $\mathbf{2 0 2 0}$}

Aunque podríamos referirnos a anteriores iniciativas de la Instituciones comunitarias ${ }^{9}$, puesto que no es nuestra intención tratar el tema que nos interesa desde su narrativa histórica, sino más bien desde una perspectiva de futuro, nos limitaremos a iniciar este epígrafe recordando la "Conclusión" de la ya citada Comunicación de la Comisión "Inteligencia artificial para Europa" de 2018:

«La UE cuenta con una sólida base industrial y científica, con universidades y laboratorios de investigación punteros, con un reconocido liderazgo en robótica, así como con empresas emergentes innovadoras. Dispone de un marco jurídico completo, que protege a los consumidores ${ }^{\mathbf{1 0}}$ al tiempo que favorece la innovación, y está avanzando en la creación de un mercado único digital. Reúne los principales ingredientes para convertirse en líder de la revolución de la IA, de acuerdo con su propia visión y basándose en sus valores.

El enfoque de la IA que se describe en el presente documento [es decir, la Comunicación de la Comisión "Inteligencia artificial para Europa" de 2018] indica el camino a seguir y destaca la necesidad de aunar fuerzas a nivel europeo, a fin de garantizar que todos los europeos formen parte de la

\footnotetext{
6 Véase: LASSE ROUHIAINEN (2018). Inteligencia artificial - 101 cosas que debes saber hoy sobre nuestro futuro, Alienta Editorial, 16-17.

7 Ibidem.

8 Véanse, inter alia: GIOVANNI SARTOR (2019) Artificial Intelligence: Challenges for EU Citizens and Consumers, European Parliament, 1-2; y GONÇALO CARRIÇO (2018) The EU and artificial intelligence: A human-centred perspective, Vol. 17 no 1, European View, 29-36.

9 La IA forma parte de la Estrategia de la Comisión para la digitalización de la industria (COM (2016) 180 final) y de la Estrategia renovada de política industrial de la UE (COM (2017) 479 final).

10 La cursiva es nuestra.
} 
transformación digital, que se dediquen recursos suficientes a la IA y que los valores y los derechos fundamentales de la Unión figuren en la vanguardia de las actividades relacionadas con ella.

Entre todos, podemos conseguir poner al servicio del progreso humano el potencial de la IA.».

En el mes de febrero de 2020, la Comisión publicó un Libro Blanco "sobre la inteligencia artificial - un enfoque europeo orientado a la excelencia y la confianza"11 en el que, tras afirmar que «la inteligencia artificial es una combinación de tecnologías que agrupa datos, algoritmos y capacidad informática», se concluía que Europa podía aunar su potencial tecnológico e industrial con una infraestructura digital de gran calidad y un marco regulador basado en sus valores fundamentales para convertirse en líder mundial de la innovación en la economía de los datos y sus aplicaciones, tal como se establece en la Estrategia Europea de Datos ${ }^{12}$. En este sentido y sobre estos cimientos, se estimaba que la Unión podía desarrollar un ecosistema de inteligencia artificial que hiciera llegar las ventajas de la tecnología a la sociedad y la economía europeas en su conjunto:

- a los ciudadanos, para que obtengan nuevos beneficios, como una mejor atención sanitaria, una menor cantidad de averías de los aparatos domésticos, unos sistemas de transporte más seguros y limpios, o mejores servicios públicos;

- al desarrollo empresarial, por ejemplo, mediante una nueva generación de productos y de servicios en áreas en las que Europa es particularmente fuerte (maquinaria, transporte, ciberseguridad, agricultura, economía verde y circular, atención sanitaria y sectores de gran valor añadido, como la moda y el turismo); y

- a los servicios de interés público, por ejemplo, mediante una reducción de los costes de la prestación de servicios (transporte, educación, energía y gestión de los residuos), una mayor sostenibilidad de los productos ${ }^{\mathbf{1 3}}$, o proporcionando a los servicios y fuerzas de seguridad las herramientas adecuadas para que aseguren la protección de los ciudadanos ${ }^{\mathbf{1 4}}$, garantizando correctamente el respeto de sus derechos y libertades.

\footnotetext{
11 COM (2020) 65 final: https://ec.europa.eu/info/sites/default/files/commission-white-paper-artificialintelligence-feb2020_es.pdf

$12 \operatorname{COM}(2020) 66$ final.

13 La inteligencia artificial y la digitalización en general son motores clave de las ambiciones contempladas en el Pacto Verde Europeo. No obstante, se estima que la huella medioambiental actual del sector de las Tecnologías de la Información y la Comunicación (TIC) se sitúa por encima del $2 \%$ del conjunto de emisiones mundiales. La Estrategia Digital Europea que acompañaba al Libro Blanco de 2020 proponía ya medidas de transformación ecológica para el sector digital.

14 Las herramientas de inteligencia artificial pueden ofrecer una oportunidad para proteger mejor a los ciudadanos de la UE de la delincuencia y los actos de terrorismo. Este tipo de herramientas podrían, por ejemplo, ayudar a detectar propaganda terrorista en línea, descubrir transacciones sospechosas en la venta de productos peligrosos, detectar objetos peligrosos ocultos o productos y sustancias ilícitos, ofrecer
} 
También en la "Introducción" del Libro Blanco se reconocía lo siguiente:

«Hoy en día, la mayor parte de los datos son relativos a los consumidores ${ }^{15}$ y se almacenan y tratan en infraestructuras ubicadas en nubes centralizadas. Frente a esto, una enorme proporción de los datos del futuro, que serán mucho más abundantes, procederá de la industria, las empresas y el sector público, y se almacenará en diversos sistemas, entre los que destacan los dispositivos informáticos que operan en el borde de la red. Este hecho ofrece nuevas oportunidades a Europa, que cuenta con una posición sólida en la industria digitalizada y las aplicaciones de comunicación empresarial, pero con una posición relativamente frágil en las plataformas de consumidores ${ }^{\mathbf{1 6}}$.

Así mismo, se hacía referencia a los consumidores en el tercer epígrafe del Libro en cuestión ("Aprovechar las próximas oportunidades: la siguiente oleada de datos") al afirmar lo que sigue:

«Aunque Europa todavía se encuentra en una posición más menos consolidada con relación a las aplicaciones de consumidores ${ }^{\mathbf{1 7}}$ y las plataformas en línea (lo que se traduce en una desventaja competitiva en el acceso a los datos), se están experimentando cambios importantes en el valor y la reutilización de los datos en los distintos sectores. El volumen de datos producido en el mundo va en aumento rápidamente, de 33 zetabytes en 2018 a una previsión de 175 zetabytes en 2025. Cada nueva oleada de datos ofrece la oportunidad a Europa de posicionarse en la economía ágil en el manejo de los datos y convertirse en líder mundial en este ámbito. Además, la manera en que se almacenan y tratan los datos cambiará drásticamente a lo largo de los próximos cinco años. A día de hoy, el $80 \%$ del tratamiento y el análisis de datos que se produce en la nube tiene lugar en centros de datos e instalaciones informáticas centralizadas, y el $20 \%$ en aparatos inteligentes conectados, como automóviles, utensilios domésticos o robots de fabricación, e instalaciones informáticas cercanas al usuario («computación en el borde»). Está previsto que, de aquí a 2025, estos porcentajes cambien de manera notable.».

Evidentemente, no podemos extendernos en el análisis del Libro Blanco "sobre la inteligencia artificial" de 2020; sí nos parece oportuno mencionar que sus redactores prestaron especial atención a:

- que un marco regulador claro para Europa generaría confianza entre los consumidores y las empresas con relación a la IA [quinto epígrafe ("Un ecosistema de confianza: el marco regulador de la IA"];

asistencia a los ciudadanos en situaciones de emergencia y servir de orientación al personal de primera intervención.

15 La cursiva es nuestra.

16 Idem.

17 Idem. 
- que los consumidores esperan el mismo nivel de seguridad y respeto de sus derechos independientemente de si un producto o un sistema está basado en la IA o no [ibidem];

- que el uso de la IA puede afectar a los valores sobre los que se fundamenta la UE y provocar la conculcación de derechos fundamentales ${ }^{\mathbf{1 8}}$, como la libertad de expresión, la libertad de reunión, la dignidad humana, la ausencia de discriminación por razón de sexo, raza u origen étnico, religión o credo, discapacidad, edad u orientación sexual, y, en su aplicación en determinados ámbitos, la protección de los datos personales y de la vida privada, el derecho a una tutela judicial efectiva y a un juicio justo, o la protección de los consumidores [ibidem, apartado "A"];

- que la UE cuenta con un marco jurídico estricto para garantizar, entre otros, la protección de los consumidores, la lucha contra las prácticas comerciales desleales y la protección de los datos personales y la privacidad [ibidem, apartado "C"]; y

- que la estructura de gobernanza de la IA debe garantizar la mayor participación de partes interesadas posible: debe consultarse a las partes interesadas (como las organizaciones de consumidores e interlocutores sociales, empresas, investigadores y organizaciones de la sociedad civil) sobre la aplicación y futuro desarrollo del marco jurídico que la regule [ibidem, apartado "H"]

En la "Conclusión" del Libro Blanco que nos ocupa no se menciona expresamente la intención de presentar una Propuesta de Reglamento por parte de la Comisión Europea; sólo se anuncia que se llevará a cabo una amplia consulta incluyendo cuestiones que estén en relación con los elementos clave para un futuro marco regulador: «esta consulta permitirá desarrollar un diálogo amplio con todas las partes interesadas que servirá de base a los siguientes pasos que dé la Comisión». Sin embargo, no cabe la menor duda de que el documento que analizaremos en el siguiente epígrafe es el resultado de las consultas llevadas a cabo según lo previsto en el mencionado Libro [que se cita en el apartado 1.1 ("Razones y objetivos de la propuesta") de la correspondiente "Exposición de motivos".

\section{La Propuesta de Reglamento sobre la IA}

Si nos atenemos a lo que la Comisión Europea ha anunciado, el nuevo Reglamento sobre la IA garantizará que los europeos puedan confiar en lo que la IA puede ofrecer. Unas reglas proporcionadas y flexibles abordarán los riesgos específicos que plantean los sistemas de IA y fijarán los estándares más altos del mundo ${ }^{\mathbf{1 9}}$. Se ha formulado

\footnotetext{
18 Según el trabajo de investigación del Consejo de Europa, un gran número de derechos fundamentales podría verse afectado por el uso de la IA (https://rm.coe.int/algorithms-and-human-rights-enrev/16807956b5).

19 Sic en https://ec.europa.eu/commission/presscorner/detail/es/ip_21_1682
} 
un plan coordinado exponiendo los cambios de política y las inversiones necesarios en los Estados miembros para reforzar el liderazgo de Europa en el fomento de una IA centrada en el ser humano (¿en el ciudadano, en el consumidor?), sostenible, segura, inclusiva y fiable 20 .

También nosotros insistiremos sobre la fiabilidad de un sistema de IA: como ha reconocido la Comisión, es preciso que las nuevas disposiciones comunitarias se apliquen directamente de la misma manera en todos los Estados miembros sobre la base de una definición de IA a prueba de futuro, por utilizar la expresión que figura en la documentación explicativa de la Propuesta de Reglamento sobre la AI.

Puesto que analizamos la Propuesta de Reglamento sobre la IA desde la perspectiva de cómo puede afectar a los consumidores la adopción de la normativa de la que se trata debemos hacer hincapié en su planteamiento basado en el riesgo, como se explica a continuación:

- Riesgo inadmisible: Se prohibirán los sistemas de IA que se consideren una clara amenaza para la seguridad, los medios de subsistencia y los derechos de las personas. Esto abarca los sistemas o las aplicaciones de IA que manipulan el comportamiento humano para eludir la voluntad de los usuarios (por ejemplo, juguetes que utilicen asistencia vocal para incitar a comportamientos peligrosos a los menores) y sistemas que permitan la «puntuación social» por parte de los Gobiernos.

- Alto riesgo: Los sistemas de IA considerados de alto riesgo abarcan las tecnologías de IA empleadas en:

- infraestructuras críticas (por ejemplo, transportes), que pueden poner en peligro la vida y la salud de los ciudadanos;

- formación educativa o profesional, que pueden determinar el acceso a la educación y la carrera profesional de una persona (por ejemplo, puntuación en exámenes);

- componentes de seguridad de los productos (por ejemplo, aplicación de IA en cirugía asistida por robots);

- empleo, gestión de trabajadores y acceso al trabajo por cuenta propia (por ejemplo, programas informáticos de clasificación de CVs para procedimientos de contratación);

20 Véase sobre la reiteración del calificativo fiable en relación con la IA: A. MAYORAL (2021). Sobre la 'fiabilidad' de la Inteligencia Artificial: BoDiA/Co (41) 7-16. 
- servicios públicos y privados esenciales (por ejemplo, sistemas de calificación crediticia que priven a los ciudadanos de la oportunidad de obtener un préstamo);

- aplicación de las leyes, que pueden interferir con los derechos fundamentales de las personas (por ejemplo, evaluación de la fiabilidad de las pruebas);

- gestión de la migración, el asilo y el control de las fronteras (por ejemplo, comprobación de la autenticidad de los documentos de viaje); y

- administración de justicia y procesos democráticos (por ejemplo, aplicación de la ley a un conjunto concreto de hechos).

Los sistemas de IA de alto riesgo estarán sujetos a obligaciones estrictas antes de que puedan comercializarse:

- sistemas adecuados de evaluación y mitigación de riesgos;

- alta calidad de los conjuntos de datos que alimentan el sistema para minimizar los riesgos y los resultados discriminatorios;

- registro de la actividad para garantizar la trazabilidad de los resultados;

- documentación detallada que aporte toda la información necesaria sobre el sistema y su finalidad para que las autoridades evalúen su conformidad;

- información clara y adecuada al usuario;

- medidas apropiadas de supervisión humana para minimizar el riesgo; y

- un alto nivel de solidez, seguridad y precisión.

Si se adopta, finalmente, el texto propuesto por la Comisión sin mayores cambios al respecto, se considerarán de alto riesgo y estarán sujetos a requisitos estrictos todos los sistemas de identificación biométrica remota ${ }^{\mathbf{2 1}}$. Su uso en directo en espacios de acceso público con fines policiales estará prohibido en principio. En la Propuesta de Reglamento sobre la IA se definen y regulan estrictamente excepciones (por ejemplo, cuando sea decididamente necesario para la búsqueda de un menor desaparecido, para prevenir una amenaza terrorista concreta e inminente, o para detectar, localizar,

21 Véanse entre las numerosas referencias bibliográficas de la que es autora LEIRE ESCAJEDO SAN EPIFANIO: (2015) El control biométrico de los ciudadanos: una nueva amenaza para los derechos fundamentales en Constitucionalismo crítico - Liber amicorum Carlos de Cabo Martín, Tirant lo Blanch: 511-540; y (2017) Tecnologías biométricas, identidad y derechos fundamentales, Editorial Aranzadi, 187 págs. (ver: https://www.academia.edu/35521202/Pólogo_Tecnologías_biométricas_identidad_y_derechos_fundame ntales). 
identificar o enjuiciar a un autor o sospechoso de un delito grave). Su uso estará sujeto a la autorización de un órgano judicial u otro organismo independiente y a los límites adecuados desde el punto de vista de la duración, el alcance geográfico y las bases de datos exploradas.

- Riesgo limitado, es decir, sistemas de IA con obligaciones específicas de transparencia: Al utilizar sistemas de IA como robots conversacionales, los usuarios deberán ser conscientes de que están interactuando con una máquina para poder tomar una decisión informada de continuar o no.

- Riesgo mínimo o nulo: En la Propuesta de Reglamento sobre la IA se prevé permitir el uso gratuito de aplicaciones tales como videojuegos basados en la IA o filtros de correo basura ${ }^{22}$; si el proyecto de Reglamento no interviene en estos supuestos es porque se considera que estos sistemas de IA solo representan un riesgo mínimo o nulo para los derechos o la seguridad de los ciudadanos ${ }^{23}$.

En términos de gobernanza, la Comisión propone que las autoridades nacionales de vigilancia del mercado controlen las nuevas reglas, mientras que la creación de un Comité Europeo de Inteligencia Artificial facilitará su aplicación e impulsará la creación de normas en materia de IA. Además, se proponen códigos de conducta voluntarios para la IA que no entrañe un alto riesgo, así como espacios controlados de pruebas para facilitar la innovación responsable.

La base jurídica de la propuesta es, en primer lugar, el artículo 114 del Tratado de Funcionamiento de la Unión Europea (TFUE), que prevé la adopción de medidas para garantizar el establecimiento y el funcionamiento del mercado interior ${ }^{\mathbf{2 4}}$. En efecto, tal como se subraya en la propia Propuesta de Reglamento sobre la IA, ésta constituye un elemento fundamental de la estrategia del mercado único digital de la UE; su objetivo principal es por lo tanto garantizar el correcto funcionamiento del mercado interior mediante el establecimiento de reglas armonizadas, en particular sobre el desarrollo, la comercialización en el mercado de la Unión y el uso de productos y servicios que utilicen tecnologías de IA o se proporcionen como sistemas independientes de IA.

En la "Exposición de motivos" de la tantas veces citada Propuesta se hace referencia a que algunos Estados miembros ya están considerando adoptar normativas nacionales para garantizar que la IA sea segura y se desarrolle y utilice de

\footnotetext{
22 Según la Comisión Europea, la inmensa mayoría de los sistemas de IA entra en esta categoría (?).

${ }^{23}$ Véase una opinión contraria (que compartimos) en: A. MAYORAL (2021). Op. cit., 8-9.

24 Véase el primer considerando del texto propuesto en el que se declara: «El objetivo del presente Reglamento es mejorar el funcionamiento del mercado interior estableciendo un marco jurídico uniforme, en particular para el desarrollo, la comercialización y el uso de la inteligencia artificial de conformidad con los valores de la Unión. El presente Reglamento persigue una serie de razones imperativas de interés público, como un alto nivel de protección de la salud, la seguridad y los derechos fundamentales, y garantiza la libre circulación transfronteriza de bienes y servicios basados en la IA, evitando así que los Estados miembros impongan restricciones al desarrollo, comercialización y uso de sistemas de inteligencia artificial, a menos que esté explícitamente autorizado por este Reglamento.» (La cursiva es nuestra).
} 
conformidad con las obligaciones de derechos fundamentales. Según la Comisión Europea este tipo de iniciativas podría dar lugar a dos problemas principales:

i. una fragmentación del mercado interior en elementos esenciales relacionados, en particular, con los requisitos para los productos y servicios de IA, su comercialización, su uso, la responsabilidad y la supervisión por parte de las autoridades públicas,

ii. y la disminución sustancial de la seguridad jurídica tanto para los proveedores como para los usuarios de los sistemas de inteligencia artificial sobre cómo se aplicarán las normas nuevas y existentes a esos sistemas en la Unión.

Dada la amplia circulación de productos y servicios a través de las fronteras, la mejor forma de resolver estos dos problemas es, siempre según la Comisión, la armonización de la legislación de la UE.

De hecho, en la propia Propuesta de Reglamento sobre la IA se definen los requisitos obligatorios comunes aplicables al diseño y desarrollo de ciertos sistemas de IA antes de su comercialización que se pondrán en funcionamiento mediante normas técnicas armonizadas. La propuesta también aborda la situación después de que los sistemas de IA se hayan introducido en el mercado armonizando la forma en que se llevan a cabo los controles ex post.

Además del ya citado artículo 114 TFUE, y teniendo en cuenta que el texto propuesto contiene determinadas disposiciones específicas sobre la protección de las personas en lo que respecta al tratamiento de datos personales ${ }^{25}$ la Comisión ha decidido basar también el futuro Reglamento, en lo que respecta a esas reglas específicas, en el artículo 16 del TFUE.

Para completar este análisis no exhaustivo de la Propuesta de Reglamento sobre la $I A$, nos parece oportuno transcribir a continuación los siguientes artículos del texto que la Comisión propone:

Artículo 1

"Objeto"

El presente Reglamento establece:

(a) reglas armonizadas para la comercialización, puesta en servicio y uso de sistemas de inteligencia artificial (sistemas de IA) en la Unión;

(b) prohibiciones de determinadas prácticas de inteligencia artificial;

25 En particular restricciones al uso de sistemas de IA para la identificación biométrica remota en "tiempo real" en espacios de acceso público con el fin de aplicación de la legislación aplicable/vigente. 
(c) requisitos específicos para los sistemas de IA de alto riesgo y obligaciones para los operadores de dichos sistemas;

(d) reglas de transparencia armonizadas para los sistemas de inteligencia artificial destinados a interactuar con personas físicas, sistemas de reconocimiento de emociones y sistemas de categorización biométrica, y sistemas de inteligencia artificial utilizados para generar o manipular contenido de imágenes, audio o video;

(e) normas sobre seguimiento y vigilancia del mercado.

Artículo 2

"Ámbito de aplicación"

1.El presente Reglamento se aplicará a:

a) proveedores que comercialicen o pongan en servicio sistemas de inteligencia artificial en la Unión, con independencia de que dichos proveedores estén establecidos en la Unión o en un tercer país;

(b) usuarios de sistemas de IA ubicados dentro de la Unión;

c) proveedores y usuarios de sistemas de inteligencia artificial ubicados en un tercer país, donde la producción producida por el sistema se utiliza en la Unión;

2. En el caso de los sistemas de IA de alto riesgo que sean componentes de seguridad de productos o sistemas, o que sean en sí mismos productos o sistemas, que entren en el ámbito de aplicación de los siguientes actos, solo se aplicará el artículo 84 del presente Reglamento ${ }^{26}$ :

a) Reglamento (CE) 300/200827;

b) Reglamento (UE) $167 / 2013^{28}$;

c) Reglamento (UE) $168 / 2013^{29}$;

(d) Directiva 2014/90/UE ${ }^{30}$;

\footnotetext{
26 Reenvío a verificar cuando se publiquen otras versiones lingüísticas.

27 Reglamento (CE) No 300/2008, de 11 de marzo de 2008, sobre normas comunes para la seguridad de la aviación civil y por el que se deroga el Reglamento (CE) No 2320/2002.

28 Reglamento (UE) No 167/2013, de 5 de febrero de 2013, relativo a la homologación de los vehículos agrícolas o forestales, y a la vigilancia del mercado de dichos vehículos.

29 Reglamento (UE) No. 168/2013, de 15 de enero de 2013, relativo a la homologación de los vehículos de dos o tres ruedas y los cuatriciclos, y a la vigilancia del mercado de dichos vehículos.

30 Directiva 2014/90/UE, de 23 de julio de 2014, sobre equipos marinos, y por la que se deroga la Directiva 96/98/CE del Consejo.
} 
e) Directiva (UE) 2016/79731;

f) Reglamento (UE) 2018/85832;

(g) Reglamento (UE) 2018/113933;

(h) Reglamento (UE) 2019/214434.

3. El presente Reglamento no se aplicará a los sistemas de inteligencia artificial desarrollados o utilizados exclusivamente con fines militares.

4. El presente Reglamento no se aplicará a las autoridades públicas de un tercer país ni a las organizaciones internacionales que entren en el ámbito de aplicación del presente Reglamento de conformidad con el apartado 1, cuando dichas autoridades u organizaciones utilicen sistemas de inteligencia artificial en el marco de acuerdos internacionales de cooperación policial y judicial. con la Unión o con uno o más Estados miembros.

5. El presente Reglamento no afectará a la aplicación de las disposiciones sobre la responsabilidad de los proveedores de servicios intermedios establecidas en el capítulo II, sección IV de la Directiva 2000/31 / CE del Parlamento Europeo y del Consejo $^{35}$ [que se sustituirá por las disposiciones correspondientes de la "Ley de Servicios Digitales" $\left.{ }^{\prime 36}\right] \gg$.

\footnotetext{
31 Directiva (UE) 2016/797, de 11 de mayo de 2016, sobre la interoperabilidad del sistema ferroviario dentro de la Unión Europea.

32 Reglamento (UE) 2018/858, de 30 de mayo de 2018, sobre la homologación y la vigilancia del mercado de los vehículos de motor y sus remolques y de los sistemas, los componentes y las unidades técnicas independientes destinados a dichos vehículos, por el que se modifican los Reglamentos (CE) No 715/2007 y (CE) No 595/2009 y por el que se deroga la Directiva 2007/46/CE.

33 Reglamento (UE) 2018/1139, de 4 de julio de 2018, sobre normas comunes en el ámbito de la aviación civil y por el que se crea una Agencia de la Unión Europea para la Seguridad Aérea y por el que se modifican los Reglamentos (CE) No 2111/2005, (CE) No 1008/2008, (UE) No 996/2010, (CE) No 376/2014 y las Directivas 2014/30/UE y 2014/53/UE del Parlamento Europeo y del Consejo y se derogan los Reglamentos (CE) No 552/2004 y (CE) No 216/2008 del Parlamento Europeo y del Consejo y el Reglamento (CEE) No 3922/91 del Consejo.

34 Reglamento (UE) 2019/2144, de 27 de noviembre de 2019 relativo a los requisitos de homologación de tipo de los vehículos de motor y de sus remolques, así como de los sistemas, componentes y unidades técnicas independientes destinados a esos vehículos, en lo que respecta a su seguridad general y a la protección de los ocupantes de los vehículos y de los usuarios vulnerables de la vía pública, por el que se modifica el Reglamento (UE) 2018/858 del Parlamento Europeo y del Consejo y se derogan los Reglamentos (CE) No 78/2009, (CE) No 79/2009 y (CE) No 661/2009 del Parlamento Europeo y del Consejo y los Reglamentos (CE) No 631/2009, (UE) No 406/2010, (UE) No 672/2010, (UE) No 1003/2010, (UE) No 1005/2010, (UE) No 1008/2010, (UE) No 1009/2010, (UE) No 19/2011, (UE) No 109/2011, (UE) No 458/2011, (UE) No 65/2012, (UE) No 130/2012, (UE) No 347/2012, (UE) No 351/2012, (UE) No $1230 / 2012$ y (UE) 2015/166 de la Comisión.

35 Directiva 2000/31/CE del Parlamento Europeo y del Consejo, de 8 de junio de 2000, relativa a determinados aspectos jurídicos de los servicios de la sociedad de la información, en particular el comercio electrónico en el mercado interior (Directiva sobre el comercio electrónico).

${ }^{36}$ https://ec.europa.eu/info/strategy/priorities-2019-2024/europe-fit-digital-age/digital-services-actensuring-safe-and-accountable-online-environment_es
} 


\section{Conclusión: fumus boni iuris}

Si en el título del epígrafe conclusivo o concluyente de nuestro artículo utilizamos esta expresión latina (que significa "apariencia de buen derecho" y es uno de los criterios aún utilizados en diversas jurisdicciones ${ }^{37}$ ) es porque, en principio, nos parece que si se adopta el texto que propone la Comisión, sin mayores cambios, se tratará de una medida que reafirmará o incluso reforzará la protección de los consumidores.

Tanto en los EE.UU. como en la Unión Europea se ha debatido sobre el enfoque que debía prevalecer al regular jurídicamente la $\mathrm{IA}^{\mathbf{3 8}}$ : ¿Debería ser restrictivo incluso hasta el punto de prohibir ciertos usos de la IA desde el principio, con el objetivo de proteger a los consumidores? ¿O debería ser menos prohibitivo para permitir la innovación facilitando a la vez a los consumidores la mayor cantidad de información posible para que éstos puedan comprender cómo funciona la IA y los datos que está utilizando y luego objetar potencialmente una decisión tomada por la IA?

La UE ha optado claramente por la primera opción. El proyecto de Reglamento establece el despliegue de la IA dentro del marco de los derechos fundamentales de la UE establecido desde hace mucho tiempo. Como hemos expuesto, se ocupa de la IA de "alto riesgo" y establece también un sistema para el registro de aplicaciones independientes de IA de alto riesgo en una base de datos pública de la UE. Los proveedores de IA deben proporcionar "información significativa" sobre los sistemas y preparar evaluaciones de conformidad. Se deduce de lo propuesto por la Comisión que ésta considera que las "evaluaciones de conformidad ex ante exhaustivas" son la solución más eficaz para resolver los problemas que pueden plantarse, teniendo en cuenta la incipiente experiencia en auditoría de IA.

En el texto que se presenta en la Propuesta de Reglamento sobre la IA se han incluido numerosos requisitos, como la evaluación de la conformidad, que son comunes a las normativas armonizadas aplicables a los productos de la UE ya existentes.

Para una gama de productos de gran difusión, desde juguetes hasta dispositivos médicos, que incorporen sistemas de IA, los fabricantes de productos serán considerados responsables de la conformidad de la IA como si ellos mismos fueran los proveedores de la misma. Es preciso tener en cuenta igualmente las importantes sanciones que podrán imponerse por incumplimiento.

Para los consumidores supone una especial salvaguardia para sus intereses el hecho de que, debido a la naturaleza horizontal del futuro Reglamento se haya previsto [apartado 2.2 de la "Exposición de Motivos"]:

\footnotetext{
37 https://dpej.rae.es/lema/fumus-boni-iuris

38 Véase: https://www.traverssmith.com/knowledge/knowledge-container/regulating-ai-which-approachwill-prevail/
} 
- una armónica coherencia con la legislación de la Unión vigente aplicable a los sectores en los que ya se utilizan o es probable que se utilicen sistemas de IA de alto riesgo en un futuro próximo;

- que se garantice también la coherencia con la Carta de los Derechos Fundamentales de la UE y la legislación secundaria existente de la Unión sobre protección de datos, protección del consumidor, no discriminación e igualdad de género; $y$

- complementar la legislación de la Unión existente sobre no discriminación con requisitos específicos que tengan como objetivo minimizar el riesgo de discriminación algorítmica, en particular en relación con el diseño y la calidad de los conjuntos de datos utilizados para el desarrollo de sistemas de inteligencia artificial complementados con obligaciones de verificación, gestión de riesgos, documentación y supervisión humana a lo largo del ciclo de vida de los sistemas de IA.

En nuestra opinión también puede calificarse de acertada la elección de un Reglamento como instrumento legal para regular la IA: según la Comisión esta elección se justifica por la necesidad de una aplicación uniforme de las nuevas reglas, como las relativas a la definición de IA, la prohibición de ciertas prácticas contraproducentes posibles en una gestión por IA y la clasificación de ciertos sistemas de IA. Sin duda alguna la aplicabilidad directa del futuro Reglamento, de conformidad con el artículo 288 del TFUE, reducirá la fragmentación jurídica y facilitará el desarrollo de un mercado único de sistemas de inteligencia artificial legales, seguros y fiables [apartado 2.4 de la "Exposición de Motivos"].

Nos parece también que, las disposiciones del texto que se propone no son excesivamente prescriptivas y dejan espacio para diferentes niveles de acción de los Estados miembros para elementos que no pongan en peligro los objetivos de la iniciativa, en particular la organización interna del sistema de vigilancia del mercado y la adopción de medidas para fomentar la innovación.

Para terminar sólo una objeción: probablemente los riesgos que implican para los consumidores ciertos usos de la IA, que abusivamente acumulan datos sobre compras, consultas, etcétera, debieran regularse con más detalle y precisión, pues constituyen un riesgo no de minimis... Quizás el Consejo y el Parlamento Europeo podrían mejorar el texto en cuestión a fin de lograr los que, en nuestra opinión, deben ser los resultados esperados:

- los proveedores de IA deben beneficiarse de un conjunto de requisitos mínimos pero claros, que creen seguridad jurídica y garanticen el acceso a todo el mercado único; 
- los usuarios de la IA deben beneficiarse de la seguridad jurídica de que los sistemas de IA de alto riesgo que adquieren cumplen con las leyes y respetan los valores europeos; y

- los consumidores deberían beneficiarse al reducir el riesgo de violaciones de su seguridad o derechos fundamentales.

\section{Bibliografía}

CARRIÇO, G.: «The EU and artificial intelligence: A human-centred perspective» Vol. 17 no 1, European View, 2018, 29-36.

ESCAJEDO SAN EPIFANIO, L.: «El control biométrico de los ciudadanos: una nueva amenaza para los derechos fundamentales» en Constitucionalismo crítico - Liber amicorum Carlos de Cabo Martín, Tirant lo Blanch, 2015, 511-540.

«Tecnologías biométricas, identidad y derechos fundamentales», Editorial Aranzadi, 2017, 187 págs.

MAYORAL, A.: «Sobre la 'fiabilidad' de la Inteligencia Artificial» no 41, BoDiA/Co, 2021, 7-16.

ROUHIAINEN, L.: «Inteligencia artificial - 101 cosas que debes saber hoy sobre nuestro futuro», Alienta Editorial, 2018, 352 págs.

SARTOR, G.: «Artificial Intelligence: Challenges for EU Citizens and Consumers». European Parliament», 2019, 9 págs, disponible en: https://www.europarl.europa.eu/RegData/etudes/BRIE/2019/631043/IPOL_BRI(20 19)631043_EN.pdf 